\title{
EVALUATION OF CHANGES IN ADIPOCYTOKINE CONCENTRATIONS AND CORRELATION BETWEEN ADIPOCYTOKINES AND BODY FAT PERCENTAGE AFTER ENDURANCE TRAINING IN OBESE GIRLS
}

original paper

( ) University School of Physical Education in Wroclaw

DOI: https://doi.org/10.5114/hm.2021.98461

\section{BAKHTYAR TARTIBIAN, MEHDI KUSHKESTANI}

Faculty of Physical Education and Sport Sciences, Allameh Tabataba’i University, Tehran, Iran

\begin{abstract}
Purpose. The purpose of the study was to evaluate changes in adipocytokine concentrations and the correlation between adipocytokines and body fat percentage after endurance training in obese girls.

Methods. This was a semi-experimental study with a pre-test and post-test design, in which 19 overweight girls aged 8-12 years were randomly selected. Anthropometric and blood indices were assessed before and after the intervention.

Results. The average leptin level $(p=0.016)$ and fat percentage $(p=0.002)$ were significantly reduced after 12 weeks of moderate-intensity endurance training, whereas the average adiponectin levels $(p=0.012)$ were significantly increased. There was a reverse correlation between adiponectin levels and BMI $(p=0.018)$, body fat percentage $(p=0.015)$, and leptin concentrations $(p=0.025)$.

Conclusions. Considering the adipocytokine improvement and body fat percentage reduction observed in this study, one can state that moderate-intensity endurance training has a role in the prevention of obesity-related diseases such as metabolic and cardiovascular disease.
\end{abstract}

Key words: aerobic, obesity, leptin, lifestyle, exercise, cardiovascular disease

\section{Introduction}

Previous studies suggest that there is a strong link between childhood obesity and adult metabolic and cardiovascular diseases [1]. We have also seen a developing trend in obesity among children in recent years [2]. In a study conducted in Iran, the prevalence of overweight and obesity in children aged 6-18 years has been estimated at $4 \%$ and $10 \%$, respectively, with regard to the national cut-offs [3]. Differences in education, economic status, nutrition, and family knowledge about fitness profits in Iran have contributed to the prevalence of overweight and obesity across children and adolescents [4].

Overweight and obesity result from excess body fat, which leads to alternations in the physiological responses among children. On the basis of recent studies, adipose tissue is known as an active metabolic organ, able to secrete various hormones, called adipokines or adipocytokines. Adipokines play a considerable role in regulating many biological processes, such as energy metabolism, inflammation, and diseases, including diabetes, metabolic syndrome, and atherosclerosis [5]. Latest studies have shown that there is a close relationship between adipose tissue-secreted hormones and obesity and related disorders [6]. Adiponectin and leptin are two adipokines mostly secreted by adipose tissue which have distinct effects on different organs, including the brain, kidney, liver, pancreas, muscle, and adipose tissue [1].

It is well established that leptin plays an important role in bone growth and maturation. Leptin effects have also been observed in hormone disorders such as hypothalamic amenorrhea and eating disorders among adolescents and young adults. Several studies indicate that there is a direct correlation between serum leptin levels and obesity [7] and it seems that increased serum leptin levels are due to leptin receptors resistance in obese subjects [1]. A crucial role of leptin in endocrine system regulation, bone density, blood pres-

Correspondence address: Mehdi Kushkestani, Faculty of Physical Education and Sport Sciences, Allameh Tabataba'i University, Piruzi Street 3,35, Tehran, 1734994133, Iran, e-mail: mehdi.kushk@gmail.com

Received: August 30, 2019

Accepted for publication: February 4, 2020

Citation: Tartibian B, Kushkestani M. Evaluation of changes in adipocytokine concentrations and correlation between adipocytokines and body fat percentage after endurance training in obese girls. Hum Mov. 2021;22(1):27-32; doi: https://doi. org/10.5114/hm.2021.98461. 


\section{HUMAN MOVEMENT}

B. Tartibian, M. Kushkestani, Exercise and adipokines in obese girls

sure, immune function, energy homeostasis, and insulin resistance has been well documented. In addition, locally, this adipokine contributes to cardiac function and vascular contractility regulation through a nitric oxide-independent mechanism, which represents a significant role of leptin in predicting cardiovascular disease. Interestingly, a study by Nehus et al. [8] showed that increased leptin serum levels in children were associated with chronic kidney diseases.

Adiponectin, a peptide hormone produced and secreted by adipocytes, is abundant in circulation and has an important physiological impact and antiatherogenic and antidiabetic properties [9]. The hormone plays a substantial role in glucose regulating, fatty acid metabolism, and energy homeostasis. Researches have demonstrated a reverse relationship between adiponectin and adipocytes. Also, adiponectin levels in obese children are lower than in lean children [10]. The crucial function of leptin in inflammatory mechanisms and dyslipidaemia is well documented. In addition, adiponectin plasma levels are reduced in metabolic diseases, including insulin resistance, type 2 diabetes, and metabolic syndrome [11]. Novel studies point at a role of this protein in preventing cardiomyopathy, atherosclerosis, and endothelial dysfunction [12].

The significance of regular exercise has been proven in the development of positive adaptations to prevent and treat obesity. In other words, exercise can lead to the modulation of metabolic hormones, reduction of chronic inflammation [13], improvement of body composition [14], and prevention of obesity-related diseases [15]. Physical activity benefits depend on the activity type, intensity, and duration [16]. Recent studies have suggested that endurance training affords physical and mental health to children and adolescents at school ages [17]. In this regard, it has been reported that moderate-intensity endurance training reduces serum leptin levels in obese girls. Garanty-Bogacka et al. [13] also found that moderate-intensity endurance training leads to an increase of adiponectin levels among obese children and adolescents.

Considering the obesity expansion, as well as the difficult conditions of research in children and adolescents, few studies have been conducted on the role of physical activity in the health and fitness of children aged 8-12 years. In addition, no study that would evaluate changes in adiponectin and leptin serum levels and body fat percentage after endurance training has been reported in Iran. Therefore, regarding the aforementioned literature and the effect of physical activity on adipose tissue-secreted hormones, we assumed that moderate-intensity endurance training led to adipokine levels modification and body fat percentage improvement. Hence, the present study investigates changes in adiponectin and leptin concentrations, as well as the correlation between these hormones and body fat percentage and body mass index (BMI) after 12 weeks of endurance training.

\section{Material and methods}

\section{Subjects and screening measurement}

The study sample involved 20 obese girls (BMI > 25) (in accordance with the Centers for Disease Control and Prevention growth charts) aged 8-12 years. They were randomly selected after primary registrations. Then they completed a questionnaire that included questions regarding personal characteristics, health,

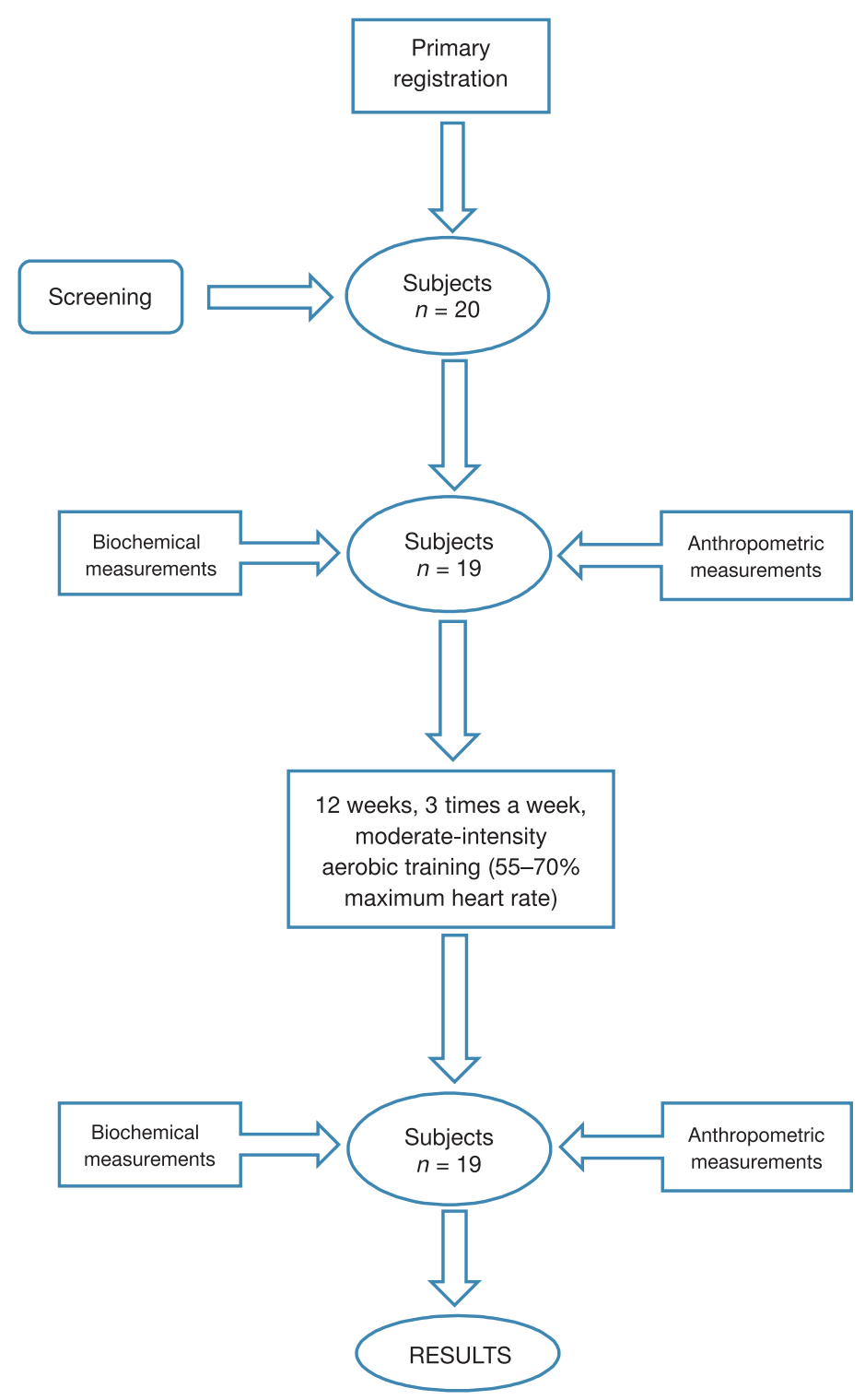

Figure 1. Subjects and study design 
and physical activity history. The criteria for participation in the current research comprised no history of hypertension, diabetes, cardiovascular disease, or any other known medical condition, no use of medical substances, no experience of regular exercise training, and no smoking. After screening, 1 participant was excluded from the research project (Figure 1). The subjects and their parents were given a detailed explanation of the study purpose and methods.

\section{Training protocol and study design}

The paper presents a quasi-experimental study with a pre-/post-intervention design. The exercise program was based on the recommendations of the American College of Sports Medicine and was reviewed by a specialist physician. First, the maximum heart rate was determined with the equation by Miller et al. [18] with $0.81 \%$ validity for obese subjects. The girls participated in a 12-week endurance training program (3 sessions per week). The program included a 15-minute warm-up consisting of jogging and stretching, followed by 30-minute endurance exercise at $55-70 \%$ of maximum heart rate (the exercise duration increased to 65 minutes at the end of the program); at the end of each session, there was a cool-down period consisting of slow running and stretching for 10 minutes. The subjects were asked to elude any physical activities that varied from their ordinary routines and not to modify their diet during the study period.

\section{Anthropometric indices measurement}

Anthropometric indices and body composition were evaluated and recorded before and after the 12 weeks of moderate-intensity endurance training. To measure weight $(\mathrm{kg})$ and height $(\mathrm{cm})$, digital scales (Seca, Germany) were used. BMI was calculated (weight in kilograms / height in meters squared), and body fat percentage was assessed with a BodyLogic 6030 body fat analyser (Omron, Korea). Blood pressure and heart rate were measured with Omron and Polar devices, respectively.

\section{Nutrition assessment}

To control and assess the individuals' nutrition status, we used a self-administered Diet History Questionnaire. It is a relatively brief food frequency questionnaire that includes a variety of foods and beverage items, as well as questions about portion sizes and dietary supplements. The questionnaire was filled in by the children's parents once a month. The validity of the questionnaire has been confirmed in the Iranian population [19].

\section{Biochemical indices measurement}

Blood samples $(3 \mathrm{ml})$ were collected from the brachial vein in the fasting condition in the morning (09:00) before and after the 12 weeks of moderate-intensity endurance training. Then, the blood samples were placed at room temperature for 10 minutes; when a blood clot formed, serum was separated from the clot by centrifugation and stored in a refrigerator at $-20^{\circ} \mathrm{C}$. Plasma leptin was measured by using an enzymelinked immunosorbent assay (ELISA) kit (EIA-2395, DRG Diagnostics, Germany). Plasma adiponectin was assessed with an enzyme-linked immunosorbent assay (ELISA) kit (K4901-100, BioVision, Germany).

\section{Data analysed}

In this study, we analysed changes in plasma leptin and adiponectin concentrations and the correlation between the levels of these adipokines and body fat percentage and BMI after 12 weeks of moderate-intensity endurance training. The statistical analysis of the data involved means and standard deviations. The Shapiro-Wilk test was performed in advance to confirm the normal distribution of each variable. Changes between the subjects' pre- and post-training biochemical and anthropometric variables were analysed with a paired $t$-test. The correlation between variables was estimated by Pearson correlation coefficients. Changes were considered statistically significant at $p<0.05$. The statistical analyses were carried out with the SPSS for Windows software (version 23, PASW Statistics SPSS, Chicago, IL, USA).

\section{Ethical approval}

The research related to human use has complied with all the relevant national regulations and institutional policies, has followed the tenets of the Declaration of Helsinki, and has been approved by the Human Subject Internal Review Board Committee of the Urmia University (Iran).

\section{Informed consent}

Informed consent has been obtained from all individuals included in this study and their parents. 


\section{HUMAN MOVEMENT}

B. Tartibian, M. Kushkestani, Exercise and adipokines in obese girls

\section{Results}

The subjects' general characteristics are summarized in Table 1. The physiological characteristics of the participants at baseline and after 12 weeks of training are presented in Table 2 . The study revealed that the average leptin level $(p=0.016)$ and fat percentage $(p=0.002)$ were significantly reduced after 12 weeks of moderate-intensity endurance training, whereas the average adiponectin levels ( $p=0.012$ ) were significantly increased. The average BMI $(p=0.115)$ did not show any significant changes (Table 2).

There was a reverse correlation between adiponectin levels and BMI ( $p=0.018)$, body fat percentage ( $p=$ $0.015)$, and leptin levels $(p=0.025)$. A positive correlation was observed between leptin and BMI $(p=0.010)$

Table 1. Basic characteristics of the subjects

\begin{tabular}{lc}
\hline Variables & Mean $\pm S D$ \\
\hline Age (years) & $9.8 \pm 1.5$ \\
Height (cm) & $140.3 \pm 7.7$ \\
Weight (kg) & $49.4 \pm 11.6$ \\
DBP (mm Hg) & $65.8 \pm 10.7$ \\
SBP (mm Hg) & $120.8 \pm 23$ \\
\hline
\end{tabular}

DBP - diastolic blood pressure

SBP - systolic blood pressure after 12 weeks of moderate-intensity endurance training (Table 3).

\section{Discussion}

The study analysed the effects of moderate-intensity endurance training on changes in plasma concentrations of adipocytokines, as well as correlations between adipocytokine plasma concentration, body fat percentage, and BMI in obese children. Our findings show that body fat percentage significantly decreased (14\%) after 12 weeks of moderate-intensity endurance training. The result was consistent with previous studies [20-22]. It seems that endurance training-induced adaptations, including increased cell respiration due to oxidative mitochondrial enzymes capacity increment and higher oxidation of fatty acids, lead to a reduction in body fat percentage in overweight children [23].

A positive correlation was observed between leptin levels and BMI after 12 weeks of moderate-intensity endurance training. This result was in agreement with that obtained by Palacios-González et al. [24].

The concentration of leptin significantly decreased (19\%) after 12 weeks of moderate-intensity endurance training, in line with a systematic review and metaanalysis by Sirico et al. [1]. Previous studies have shown that overweight and obesity increase leptin receptor

Table 2. Biochemical and anthropometric indices at baseline and after the 12 -week training $($ mean $\pm S D)$

\begin{tabular}{lccc}
\hline Parameter & Before training & After training & $p$ \\
\hline Leptin $(\mathrm{ng} / \mathrm{ml})$ & $6.7 \pm 1.9$ & $5.4 \pm 2^{*}$ & 0.016 \\
Adiponectin $(\mu \mathrm{g} / \mathrm{ml})$ & $7.56 \pm 2.52$ & $16.08 \pm 4.84^{*}$ & 0.012 \\
Body mass index $\left(\mathrm{kg} / \mathrm{m}^{2}\right)$ & $27.2 \pm 4$ & $24.8 \pm 3.8$ & 0.115 \\
Fat $(\%)$ & $34.2 \pm 5.3$ & $29.4 \pm 4.4^{* *}$ & 0.002 \\
\hline
\end{tabular}

${ }^{*} p<0.05,{ }^{*} p<0.01$

Table 3. Correlation between blood chemistry and anthropometric data

\begin{tabular}{lccccc}
\hline Parameter & Coefficient & Leptin changes & $\begin{array}{c}\text { Adiponectin } \\
\text { changes }\end{array}$ & $\begin{array}{c}\text { Body mass index } \\
\text { changes }\end{array}$ & Fat (\%) changes \\
\hline \multirow{2}{*}{ Leptin $(\mathrm{ng} / \mathrm{ml})$} & $r$ & - & -0.621 & 0.577 & 0.108 \\
& $p$ & - & $0.025^{*}$ & $0.010^{*}$ & 0.661 \\
Adiponectin $(\mu \mathrm{g} / \mathrm{ml})$ & $r$ & -0.621 & - & -0.741 & -0.612 \\
& $p$ & $0.025^{*}$ & - & $0.018^{*}$ & $0.015^{*}$ \\
Body mass index $\left(\mathrm{kg} / \mathrm{m}^{2}\right)$ & $r$ & 0.577 & -0.741 & - & 0.163 \\
& $p$ & $0.010^{*}$ & $0.018^{*}$ & - & 0.505 \\
Fat $(\%)$ & $r$ & 0.108 & -0.612 & 0.163 & - \\
& $p$ & 0.661 & $0.015^{*}$ & 0.505 & - \\
\hline
\end{tabular}

${ }^{*} p<0.05$ 
resistance, raises serum leptin circulation levels [4]. In the context of this mechanism, one can possibly state that moderate-intensity endurance training can lead to a reduction and modulation of leptin serum levels in children by influencing the overexpression of leptin receptors and improving its function [25]. On the other hand, with the consideration of the positive relationship between serum leptin levels and body fat percentage [26], it can be indicated that endurance training reduces leptin serum levels by declining obesity.

It is also our finding that there is a negative correlation between adiponectin levels and body fat percentage and BMI after 12 weeks of moderate-intensity endurance training. This corroborates a study by Jeon et al. [27]. We observed that adiponectin concentrations significantly increased (112\%) after 12 weeks of moderate-intensity endurance training, which is in line with a study by Linares-Segovia et al. [28], a systematic review and meta-analysis by García-Hermoso et al. [5], and a paper by Sirico et al. [1]. Adipose tissue is known as an organ that produces adiponectin and secretes it into the systemic circulation. On the other hand, previous evidence along with this study suggest a reverse relationship between body fat percentage and adiponectin levels: overweight and obesity result in a reduction of adiponectin serum concentrations. In accordance with the results, one can state that moderate-intensity endurance training decreases body fat percentage, modifies adipose tissue hormones, and increases adiponectin secretion.

In the presented study, BMI did not significantly decrease (5\%) after 12 weeks of moderate-intensity endurance training. This result conforms with that obtained by Santos Silva et al. [29]. Since changes in BMI are associated with body growth and muscle mass, BMI alone would not be the best way to assess the effects of physical changes after exercise intervention in children and adolescents [29]. Thus, considering the results of the present and previous studies, we can assert that moderate-intensity endurance training can play a vital role in improving the body composition of overweight children.

\section{Limitation}

In the context of the age of the subjects in this study and puberty-induced physiological changes, the absence of a control group was an important limitation. Also, owing to the biological differences in this age group (8-12 years), especially among girls, further studies investigating this age range are recommended.

\section{Conclusions}

Generally, considering the adipocytokine improvement and body fat percentage reduction observed in this study, one can state that moderate-intensity endurance training has a role in the prevention of obesity-related diseases such as metabolic and cardiovascular disease.

\section{Acknowledgments}

The authors are grateful to the subjects who participated in the study.

\section{Disclosure statement}

No author has any financial interest or received any financial benefit from this research.

\section{Conflict of interest}

The authors state no conflict of interest.

\section{References}

1. Sirico F, Bianco A, D’Alicandro G, Castaldo C, Montagnani S, Spera R, et al. Effects of physical exercise on adiponectin, leptin, and inflammatory markers in childhood obesity: systematic review and meta-analysis. Child Obes. 2018;14(4):207-217; doi: 10.1089/ chi.2017.0269.

2. Pelegrini A, Pinto ADA, Petroski EL. Agreement between body mass index and the sum of skinfolds in adolescents with different levels of physical activity. Hum Mov. 2018;19(2):11-17; doi: 10.5114/hm.2018.74056.

3. Kelishadi R, Ardalan G, Gheiratmand R, Majdzadeh R, Hosseini M, Gouya MM, et al. Thinness, overweight and obesity in a national sample of Iranian children and adolescents: CASPIAN study. Child Care Health Dev. 2008;34(1):44-54; doi: 10.1111/j.1365-2214.2007. 00744.x.

4. Mirmohammadi S-J, Hafezi R, Mehrparvar AH, Rezaeian B, Akbari H. Prevalence of overweight and obesity among Iranian school children in different ethnicities. Iran J Pediatr. 2011;21(4):514-520.

5. García-Hermoso A, Ceballos-Ceballos RJM, PobleteAro CE, Hackney AC, Mota J, Ramírez-Vélez R. Exercise, adipokines and pediatric obesity: a meta-analysis of randomized controlled trials. Int J Obes. 2017;41(4): 475-482; doi: 10.1038/ijo.2016.230.

6. Jaleel A, Aheed B, Jaleel S, Majeed R, Zuberi A, Khan S, et al. Association of adipokines with obesity in children and adolescents. Biomark Med. 2013;7(5):731-735; doi: $10.2217 /$ bmm.13.41.

7. Jmal A, Bouyahya O, Ayadi I, Occhi H, Feki M, Kaabachi N, et al. Serum leptin concentration in Tunisian non obese children. Ann Biol Clin. 2010;68(3):311-315; doi: 10.1684/abc.2010.0433. 
B. Tartibian, M. Kushkestani, Exercise and adipokines in obese girls

8. Nehus E, Furth S, Warady B, Mitsnefes M. Correlates of leptin in children with chronic kidney disease. J Pediatr. 2014;165(4):825-829; doi: 10.1016/j.jpeds.2014. 06.030 .

9. Achari AE, Jain SK. Adiponectin, a therapeutic target for obesity, diabetes, and endothelial dysfunction. Int $\mathrm{J}$ Mol Sci. 2017;18(6):1321; doi: 10.3390/ijms18061321.

10. Arrigo T, Gitto E, Ferraù V, Munafò C, Alibrandi A, Marseglia GL, et al. Effect of weight reduction on leptin, total ghrelin and obestatin concentrations in prepubertal children. J Biol Regul Homeost Agents. 2012;26 (1 Suppl.):S95-S103.

11. Klünder-Klünder M, Flores-Huerta S, García-Macedo R, Peralta-Romero J, Cruz M. Adiponectin in eutrophic and obese children as a biomarker to predict metabolic syndrome and each of its components. BMC Public Health. 2013;13(1):88; doi: 10.1186/1471-245813-88.

12. Katsiki N, Mantzoros C, Mikhailidis DP. Adiponectin, lipids and atherosclerosis. Curr Opin Lipidol. 2017; 28(4):347-354; doi: 10.1097/mol.0000000000000431.

13. Garanty-Bogacka B, Rać M, Syrenicz M, Gębala A, Walczak M, Syrenicz A. Changes in serum adipocytokines and inflammatory biomarkers following oneyear of exercise training in obese adolescents. J Diabetes Metab.2012;3(7):212; doi:10.4172/2155-6156.1000212.

14. Arciero PJ, Baur D, Connelly S, Ormsbee MJ. Timeddaily ingestion of whey protein and exercise training reduces visceral adipose tissue mass and improves insulin resistance: the PRISE study. J Appl Physiol. 2014; 117(1):1-10; doi: 10.1152/japplphysiol.00152.2014.

15. Golbidi S, Laher I. Exercise induced adipokine changes and the metabolic syndrome. J Diabetes Res. 2014; 2014:726861; doi: 10.1155/2014/726861.

16. Bouassida A, Chamari K, Zaouali M, Feki Y, Zbidi A, Tabka Z. Review on leptin and adiponectin responses and adaptations to acute and chronic exercise. Br J Sports Med. 2010;44(9):620-630; doi: 10.1136/bjsm. 2008.046151.

17. Nadeau KJ, Maahs DM, Daniels SR, Eckel RH. Childhood obesity and cardiovascular disease: links and prevention strategies. Nat Rev Cardiol. 2011;8(9):513525; doi: 10.1038/nrcardio.2011.86.

18. Miller WC, Wallace JP, Eggert KE. Predicting max HR and the $\mathrm{HR}-\mathrm{VO}_{2}$ relationship for exercise prescription in obesity. Med Sci Sports Exerc. 1993;25(9):1077-1081.

19. Toorang F, Sasanfar B, Razeghi Jahromi S, Ebrahimpour Koujan S, Narmcheshm S, Rafei A, et al. Validation of Diet History Questionnaire in assessing energy and nutrient intakes of Iranian population. Iran J Public Health. 2019;48(6):1074-1081; doi: 10.18502/ ijph.v48i6.2906.

20. Kelley GA, Kelley KS. Effects of exercise in the treatment of overweight and obese children and adolescents: a systematic review of meta-analyses. J Obes. 2013; 2013:783103; doi: 10.1155/2013/783103.
21. Contrò V, Bianco A, Cooper J, Sacco A, Macchiarella A, Traina M, et al. Effects of different circuit training protocols on body mass, fat mass and blood parameters in overweight adults. J Biol Res. 2017;90:6279; doi: 10.4081/jbr.2017.6279.

22. Maciaszek J, Skrypnik D, Ratajczak M, Stemplewski R, Osiński W, Bogdański P, et al. Two aerobic exercise programs in management of back pain among middle-aged obese women: a randomized controlled study. Hum Mov. 2016;17(2):72-79; doi: 10.1515/humo-2016-0016.

23. Lundby C, Jacobs RA. Adaptations of skeletal muscle mitochondria to exercise training. Exp Physiol. 2016; 101(1):17-22; doi: 10.1113/EP085319.

24. Palacios-González B, Vadillo-Ortega F, Polo-Oteyza E, Sánchez T, Ancira-Moreno M, Romero-Hidalgo S, et al. Irisin levels before and after physical activity among school-age children with different BMI: a direct relation with leptin. Obesity. 2015;23(4):729-732; doi: 10.1002/oby.21029.

25. Izwan TOS, Sarawati MNVN, Hidayah AZN, Afifurrahman JM, Farah NFM, Appukutty M, et al. Expression of leptin-receptor (LEP-R) and angiotensin-converting enzyme (ACE) genes after a 12-week high intensity interval training (HIIT) intervention among obese individuals. J Fundam Appl Sci. 2018;10(6S): 752-765; doi: 10.4314/jfas.v10i6s.30.

26. Reinehr T, Roth C, Menke T, Andler W. Adiponectin before and after weight loss in obese children. J Clin Endocrinol Metab. 2004;89(8):3790-3794; doi: 10.1210/ jc.2003-031925.

27. Jeon J-Y, Han J, Kim H-J, Park MS, Seo DY, Kwak Y-S. The combined effects of physical exercise training and detraining on adiponectin in overweight and obese children. Integr Med Res. 2013;2(4):145-150; doi: 10.1016/j.imr.2013.10.001.

28. Linares-Segovia B, Guízar-Mendoza JM, Amador-Licona N, Barbosa-Sabanero G, Malacara JM. Effect of an exercise program, on hemodynamic, metabolic and inflammatory markers in obese Mexican adolescents. Endocrinol Metab Synd. 2013;S2:003; doi: 10.4172/ 2161-1017.S2-003.

29. Santos Silva DA, Petroski EL, Pelegrini A. Effects of aerobic exercise on the body composition and lipid profile of overweight adolescents. Rev Bras Ciênc Esporte. 2014;36(2):295-309; doi: 10.1590/S0101-328920140 00200002 . 